\title{
Evaluation of the Therapeutic Effectiveness of Polymyxin B Versus Colistin in Carbapenem-resistanct Klebsiella pneumoniae-Associated Bacteremia
}

\author{
Md. J ahidul Hasan ${ }^{1}$, Raihan Rabbani ${ }^{2}$ and Sitesh C. Bachar ${ }^{3}$ \\ ${ }^{1}$ Clinical Pharmacy Services, Department of Pharmacy, Square Hospitals Ltd., Dhaka, Bangladesh \\ ${ }^{2}$ Internal Medicine and ICU, Square Hospitals Ltd., Dhaka, Bangladesh \\ ${ }^{3}$ Department of Pharmacy, University of Dhaka, Bangladesh
}

(Received: February 25, 2019; Accepted: June 19, 2019; Published (Web): October 5, 2019)

\begin{abstract}
Polymyxins are the last resort antibiotics for treating carbapenem-resistance Klebsiella pneumoniae $(\mathrm{CR}-K p$ )-associated bacteremia. The main aim of this study was to demonstrate the therapeutic effectiveness of polymyxin B versus colistin in the treatment of CR-Kp-associated Bacteremia. This 12-month long cross-sectional study was conducted on 54 and 47 patients suffering from CR-Kp-associated Bacteremia, and residing into two separate groups, they were treated with polymyxin B and colistin, respectively. After 5 day-treatment, the microbiological eradication rate was found $96.30 \%(52 ; n=54)$ in polymyxin B group and $87.23 \%(41 ; n=47)$ in colistin group. In the 30-day mortality rate-comparison, $51.03 \%$ more death was found in colistin group $(34.04 \%, \mathrm{n}=$ 47) than the polymyxin B group $(16.67 \%, \mathrm{n}=54)$. In this study, Polymyxin B showed superior therapeutic potentiality with less number of secondary infections and 30-day mortality rate in CR-Kp-associated bacteremia than colistin.
\end{abstract}

Key words: Carbapenem-resistance, Klebsiella pneumoniae, bacteremia, polymyxin B, colistin.

\section{INTRODUCTION}

Polymyxins are polypeptide antibiotics discovered from Paenibacillus polymyxa through fermentation, in 1947. Chemically, they are pentacationic polypeptides and five (A, B, C, D and E) different chemical compounds are collectively known as polymyxins. ${ }^{1}$ Globally, both polymyxin B (PMB) and colistin (CLN) (also known as, polymyxin E) clinically represent the polymyxins group of antibiotics, since their invention and colistin is commercially available in the form of Colistimethate Sodium (CMS) which is a pro-drug. ${ }^{2}$ Structurally, in the $6^{\text {th }}$ position of the chemical chain (starting from the $\mathrm{N}$-terminal), a phenylalanine and a D-leucine gives the chemical identity of PMB and CLN, respectively. ${ }^{3}$ After commercially launching polymyxins in global market, initially the clinical

Correspondence to: Md. Jahidul Hasan

E-mail: jahidrj@gmail.com

Mobile: +8801911011167

Dhaka Univ. J. Pharm. Sci. 18(2): 209-215, 2019 (December) DOI: https://doi.org/10.3329/dujps.v18i2.43263 
pneumoniae carbapenemase) came in the global concern with its special resistance-building mechanism, in the United States, Southern America and Europe. In Asia, first KPC was reported in China in 2007, and then in South Korea and Taiwan, subsequently. ${ }^{4}$ The mechanism of how Klebsiella pneumoniae shows resistance towards carbapenems is dependent on the production of carbapenemhydrolyzing $\beta$-lactamase, and this mechanism is also found among other enterobacteriaceae (Pseudomonas spp. and Acinetobacter spp.). ${ }^{5}$ Recently, World Health Organization (WHO) has declared this emergency of MDR enterobacteriaceae as one of the three major threats to the global human health. ${ }^{6}$ Among the currently available all antibiotic resources against these life threatening superbugs, polymyxins are considered as one of the potent therapeutic options, worldwide, and US Centers for Disease Control and Prevention (CDC) declared polymyxins as the last resort as well as the reserve group antibiotics during this crisis moment. ${ }^{7}$

Sepsis is a life threatening condition with a presence of systemic inflammatory response syndrome (SIRS) that includes mostly, bloodstream infection (BSI), respiratory tract infection, intraabdominal infection, and urinary tract infection. Bacteremia, also known as bloodstream infection, is a serious condition where a bacterium enters in to the bloodstream and effective treatment options with available antibiotics are very limited especially when, CR-Kp-associated bacteremia developes. ${ }^{8,9}$ As the last resort, both polymyxin B and colistin are the common options for treating bacteremia specially, when the organism like, Klebsiella pneumoniae is a MDR-pathogen and only sensitive to polymyxins. ${ }^{2,4}$ The main objective of this study was to observe the therapeutic effectiveness as well as clinical outcomes of the polymyxin B in comparison to colistin in the treatment of CR- $K p$-associated bacteremia among the adult critically ill patients of intensive care unit (ICU).

\section{MATERIALS AND METHODS}

This prospective cross-sectional study was done in the ICU setup of a tertiary level hospital in Dhaka,
Bangladesh and conducted continuously for 12 months (June, 2017 to May, 2018). During this period, total 1,411 patients were admitted in the ICU department came either from outside of the hospital or from the in-patient wards, with the diagnosis of having one or multiple symptoms of sepsis. As per the ICU-protocol, immediately after getting admission, their blood, tracheal aspirates/sputum and urine samples were sent to the microbiology laboratory for testing antibiotics' susceptibility to microorganisms (culture sensitivity) through determining the minimum inhibitory concentration (MIC). The sensitivity testing methodology (broth dilution) and equipments (BD Phoenix ${ }^{\mathrm{TM}}$ M50 Automated Microbiology System) of that microbiology lab were validated with the standards of Clinical and Laboratory Standards Institute (CLSI), United States of America. After getting the susceptibility (culture sensitivity) results with corresponding MIC values of particular antibiotics, 143 patients were found to contain CR-K. pneumoniae (MRD-organism; only sensitive to $\mathrm{PMB}$ and CLN) in their blood samples, while no other gram-negative bacteria simultaneously were found in any other tested biological samples. For those 143 patients, the only available sensitive antibiotic option was PMB and CLN (as per the individual culture sensitivity report). On the basis of the patients' age contrapositions, 119 middle-aged patients (45 to 65 years) were considered for the study purpose. Among those 119 patients, 7 patients and 11 patients in PMB and CLN groups, respectively, were excluded from the study because of death, discharge against medical advice and shifted to another hospital. Finally, the study was completed with $101(\mathrm{~N})$ patients. Among them $(\mathrm{N}=101), 54$ patients in the PMB group were treated with PMB as mono antibiotic therapy (dose: $2.5 \mathrm{mg} / \mathrm{Kg}$ body weight as loading dose, then 1-1.5 $\mathrm{mg} / \mathrm{Kg}$ body weight/day divided into 3 doses as maintenance dose) and 47 patients in the CLN group were treated with CLN, (in the form of CMS), as mono antibiotic therapy (dose of CMS: 0.17 Million IU/Kg body weight as loading dose then, maximum 9 Million/day in divided doses as maintenance dose); The selected patients $(\mathrm{N}=101)$ were distributed prior 
to initiation of the antibiotic therapies (PMB and CLN) into PMB and CLN groups as one after the other patient (sampling method of the study). Dose adjustment of antibiotics (PMB and CLN) in renal impaired patients of both the groups was done as per the hospital's antibiotic dosing protocol. The $2^{\text {nd }}$ version of the Acute Physiologic Assessment and Chronic Health Evaluation II (APACHE II) scoring system was used in this study to measure the severity of diseases in the patients of both the groups. The ethical approval for this study was taken from the Square hospital ethical committee on April, 2017.

The antibiogram (2017) of that hospital was considered for the study. All the patients of each group were treated with the group-specific antibiotic (PMB or CLN) and targeted duration of the treatment was 14 days. No patient of both groups previously had any history of CRE-infection as well as PMB or CLN-based treatment experience for any type of infections. After 5 days of initiation of the treatment with the corresponding group-wise antibiotics, thorough review by culture sensitivity (CS) tests were conducted for every patient of both groups and patients were observed for eradication rate of CR-Kp in blood and occurrences of secondary infections in other tissues (as per the CS reports) in both the groups. A 30-day mortality rate was estimated for both the groups and compared one group to another to analyze the difference. IBM SPSS statistics (version 22) software was used for this study for the orientation of all study data.

\section{RESULTS AND DISCUSSION}

The median age of PMB group $(\mathrm{n}=54)$ and CLN group $(\mathrm{n}=47)$ was 53 years and 56 years, respectively (Table 1). In both the groups, the number of male patients was higher than female patients (male and female in CLN group: 26/21 and PMB group: 29/25, respectively) (Table 1). The procalcitonin level is an infection marker and determines the severity of infection. This median procalcitonin level in CLN group was $56.28 \mathrm{ng} / \mathrm{mL}$ and that was slightly higher than PMB group (median value: $47.96 \mathrm{ng} / \mathrm{ml}$ ) (Table 1). Apache II Scoring system is the most common tool for assessing the severity of the disease specially, when the patient is in the critical areas. The minimum and the maximum range of this score in the both the groups were closer to each other (CLN group: 8.0-28.0 and PMB group: 8.0-30.0).

Table 1. Demographic characteristics of patients and infection markers.

\begin{tabular}{|c|c|c|}
\hline \multirow[b]{2}{*}{ Characteristics } & \multicolumn{2}{|c|}{ Variables } \\
\hline & $\begin{array}{l}\text { CLN group } \\
\quad(n=47)\end{array}$ & $\begin{array}{l}\text { PMB group } \\
\quad(\mathrm{n}=54)\end{array}$ \\
\hline \multicolumn{3}{|l|}{ Age (year) } \\
\hline Median & 53.0 & 56.0 \\
\hline Range (min-max) & $47-61$ & $45-63$ \\
\hline \multicolumn{3}{|l|}{ Gender } \\
\hline Male & 26 & 29 \\
\hline Female & 21 & 25 \\
\hline \multicolumn{3}{|c|}{ Procalcitonin $(<0.1$ ng/ml $)$} \\
\hline Median & 56.28 & 47.96 \\
\hline Range (min-max) & $10.60-119.30$ & $13.28-107.50$ \\
\hline \multicolumn{3}{|c|}{ Apache II Score (0-71 points) } \\
\hline Median & 15.0 & 18.0 \\
\hline Range (min-max) & $8.0-28.0$ & $8.0-30.0$ \\
\hline
\end{tabular}


No patient of both the groups (PMB and CLN) had any CRE-infection before the study period and had never been treated with the PMB or CLN for any infection purpose (Table 2). The hospital's antibiogram report showed that among 100 infectedpatients in that hospital, the sensitivity of PMB and CLN to gram-negative bacteria was $98 \%$ and $96 \%$, respectively (Table 2).

Table 2. Patients and pre-therapeutic information.

\begin{tabular}{cccc}
\hline Group & $\begin{array}{c}\text { Previous } \\
\text { history of } \\
\text { CRE- } \\
\text { infection }\end{array}$ & $\begin{array}{c}\text { Previous } \\
\text { history of } \\
\text { taking } \\
\text { PMB/CLN }\end{array}$ & $\begin{array}{c}\text { Sensitivity of } \\
\text { PMB/CLN in } \\
\text { hospital } \\
\text { antibiogram (\%) }\end{array}$ \\
\hline $\begin{array}{c}\text { PMB } \\
(\mathrm{n}=54)\end{array}$ & none & none & 98 \\
$\begin{array}{c}\text { CLN } \\
(\mathrm{n}=47)\end{array}$ & none & none & 96 \\
\hline
\end{tabular}

In PMB group and CLN group, $68.52 \%$ patients $(37 ; \mathrm{n}=54)$ and $72.34 \%$ patients $(34 ; \mathrm{n}=47)$ respectively, were not previously experienced with carbapenems (meropenem and doripenem) before the study period (Table 3). As per the study method, blood samples were analyzed and CR- $K p$ was detected in every sample of the both groups' patients, where MIC of meropenem in every CS report showed that $K$. pneumoniae (CR- $K p$ ) was resistant (> 4 $\mathrm{mcg} / \mathrm{ml}$ ) to meropenem and only sensitive to polymyxins (colistin and polymyxin $\mathrm{B})(\mathrm{MIC}=<2$ $\mathrm{mcg} / \mathrm{ml}$ ) (Table 3).

After getting the CS reports of all patients ( $\mathrm{n}=$ 101) of the study, the desired antibiotic therapy (PMB or CLN) was started, immediately. The dose and frequency of the antibiotic was started according to the hospitalized dosing recommendations, and the drug was given intravenously. For renal impaired patients, dose of the antibiotics were adjusted according to the hospital's dosing protocol as well. After 5 days of the initiation of the antibiotic therapy (PMB/CLN), in both groups, again all the biological samples including blood samples of all patients $(n=$ 101) were tested for determining the outcomes of the antibiotic therapies. In the PMB group, 52 patients

Table 3. MIC, culture sensitivity and therapeutic information.

\begin{tabular}{lccccc}
\hline Group & $\begin{array}{c}\text { Patients had no previous } \\
\text { history of getting car. } \\
(\mathrm{n}, \%)\end{array}$ & $\begin{array}{c}\text { Biological } \\
\text { sample taken } \\
\text { for CS }\end{array}$ & $\begin{array}{c}\text { Gram-negative } \\
\text { microorganism } \\
\text { Identified }\end{array}$ & $\begin{array}{c}\text { MIC of mero. with } \\
\text { sensitivity in the report }\end{array}$ & $\begin{array}{c}\text { MIC of polymyxins } \\
\text { with sensitivity }\end{array}$ \\
\hline PMB & $37(68.52)(\mathrm{n}=54)$ & Blood & CR-Kp & $\geq 4 \mathrm{mcg} / \mathrm{ml}\left(\mathrm{R}^{\mathrm{b}}\right)$ & $\leq 2 \mathrm{mcg} / \mathrm{ml}\left(\mathrm{S}^{\mathrm{c}}\right)$ \\
\hline CLN & $34(72.34)(\mathrm{n}=47)$ & Blood & CR-Kp & $\geq 4 \mathrm{mcg} / \mathrm{ml}(\mathrm{R})$ & $\leq 2 \mathrm{mcg} / \mathrm{ml}(\mathrm{S})$ \\
\hline
\end{tabular}

car $^{\mathrm{a}}$ : carbapenems; $\mathrm{R}^{\mathrm{b}}$ : resistant; $\mathrm{S}^{\mathrm{c}}$ : sensitive.

$(96.3 \%, \mathrm{n}=54)$ were found with no further presence of $K$. pneumoniae (CR-Kp) in their blood samples, and in the CLN group, 41 patients $(87.23 \%, n=47)$ were found with no $K$. pneumonia $(\mathrm{CR}-\mathrm{Kp})$ in their blood samples (Table 4). Though CR-Kp was completely eradicated from the blood of most of the patients of both groups, according to the reviewed CS reports, 4 patients $(n=54)$ were found with secondary infection in lungs with CR-Kp (detected in tracheal aspirate samples) in the PMB group whereas 15 patients were experienced with further secondary infection in lungs and wound with CR-Kp (detected in tracheal aspirates and wound swabs) in CLN group (Table 4). As per the 30-day mortality rate calculation (from the first day of initiation of the antibiotic therapy to the next 30 days, is considered as 30-day mortality period), 9 patients $(16.67 \%$; $n=$ 54) of PMB group expired; whereas 16 patients $(34.04 \%, n=47)$ died in the CLN group (Table 4). The 30-day mortality rate-comparison in between the two groups (PMB and CLN) showed that $51.03 \%$ more patients were died in the CLN group $(n=47)$ than the PMB group $(n=54)$ (Table 4). 
Table 4. Therapeutic outcomes, Secondary infection and 30-day mortality rate

\begin{tabular}{llllllll}
\hline Group & $\begin{array}{l}\text { Antibiotic } \\
\text { (PMB/CLN) } \\
\text { therapy started }\end{array}$ & $\begin{array}{l}\text { Dose, } \\
\text { frequency } \\
\text { and ROA }\end{array}$ & $\begin{array}{l}\text { Review of } \\
\text { blood CS }\end{array}$ & $\begin{array}{l}\text { No organism } \\
\text { found in CS-blood } \\
\text { review n, } \%\end{array}$ & $\begin{array}{l}\text { Secondary } \\
\text { infection with } \\
\text { CR-Kp }\end{array}$ & $\begin{array}{l}\text { 30-day } \\
\text { mortality } \\
\text { rate (\%) }\end{array}$ & $\begin{array}{l}\text { 30-day } \\
\text { mortality rate- } \\
\text { comparison }\end{array}$ \\
\hline $\begin{array}{l}\text { PMB } \\
(\mathrm{n}=54)\end{array}$ & Immediately & $\begin{array}{l}\text { As per } \\
\text { recmd., IV }\end{array}$ & 5 days later & $52(96.30)$ & 4 (in lung) & $16.67(9)$ & $\begin{array}{l}51.03 \% \text { more } \\
\text { death in CLN }\end{array}$ \\
\hline $\begin{array}{l}\text { CLN } \\
(\mathrm{n}=47)\end{array}$ & Immediately & $\begin{array}{l}\text { As per } \\
\text { recmd., IV }\end{array}$ & 5 days later & $41(87.23)$ & $\begin{array}{l}15(12: \text { lung; } \\
\left.3: \mathrm{W} / \mathrm{S}^{\mathrm{f}}\right)\end{array}$ & $34.04(16)$ & group \\
\hline
\end{tabular}

ROA $^{\mathrm{d}}$ : route of administration; Recmd. ${ }^{\mathrm{e}}$ : recommendation; $\mathrm{W} / \mathrm{S}^{\mathrm{f}}$ : wound swab.

Among the very few remaining potential antibiotics used in bacteremia caused by MDR pathogen CR- $K p$, polymyxins are nowadays the most preferable last-line option of treatment. ${ }^{9}$ Our study showed the relative therapeutic outcomes of using PMB and CLN in CR- $K p$-associated bacteremia and relatively better therapeutic outcome observed in PMB group. The endemicity of spreading MDR gram-negative organisms like, CR- $K p$ is horrific throughout the world and this fast resistancedisseminating trend is now in a word 'invincible'. In Italy, approximately $25 \%-50 \%$ of all $\mathrm{K}$. pneumoniae isolates in bloodstream is ESBL type and 20\%-30\% of strains are capable of producing Klebsiella pneumoniae carbapenemase- 2 and $-3 .{ }^{22}$ This outbreak of CR-Kp is same in other countries like, in Asia (e.g. China), South America, Southern Europe and so on. ${ }^{4}$ To fight against such superbugs, no clinical evidenced-based potential treatment option is still established in the world specially, any specific treatment guideline for treating bacteremia especially with $C R-K p .^{10}$ Even in United States, no big randomized clinical trial is still available to evaluate the comparative therapeutic-outcome among three most widely used last resort antibiotics, tigecycline, PMB and CLN. ${ }^{7,9}$ Due to limited clinical data on relative therapeutic effectiveness of polymyxins, one surveillance report found that MDR-bugs are enormously produced due to inappropriate use of last resort antibiotics including polymyxins by the physicians. ${ }^{11}$ Both irrational use of antibiotics and wrong dose adjustments during renal impairment in critically ill patients mostly lead to ultimate therapeutic failure and increase the risk of developing antibiotic resistance. ${ }^{12}$ On the basis of relative-good therapeutic outcome, a right antibiotic in the right type of infection with targeted pathogen is the appropriate attempt during this alarming superbugsera. In our study, PMB alone was found superior to CLN monotherapy in CR- $K p$-associated bacteremia management and after 5 days of treatment with PMB showed relatively higher pathogen eradication rate (PMB: $96.30 \%, n=54$ whereas CLN: $87.23 \%, n=47$ ) (Table 4).

Concerning the current pharmacokinetic and pharmacodynamic properties, CLN is accumulated in the renal system whereas PMB is accumulated in the blood stream with higher proportion. ${ }^{13}$ Multiple studies showed that following intravenous administration in healthy renal patients, CLN shows variable serum-drug concentration in regards of time because of high elimination rate of CMS (as prodrug) through the kidneys with short serum half-life whereas, PMB maintains a sustained serum-drug level in patients with both sound and impaired renal function because of the direct administration of its active form. ${ }^{14-17} \mathrm{~A}$ multi-center phase-II clinical study found that $78.1 \%$ severe blood stream infection with extensively drug-resistant (XDR) gram-negative bacteria were treated with $\mathrm{PMB}$, successfully. ${ }^{18}$ In our study, $96.3 \%(\mathrm{n}=54)$ infection with $\mathrm{CR}-K p$ were completely treated with PMB whereas CLN showed relatively less $(87.23 \%, \mathrm{n}=47)$ recovery from $\mathrm{CR}$ $K p$-associated Bloodstream infection (BSI). A prospective cohort study showed that $80 \%$ cure rate was attributed with CLN monotherapy in urinary tract infection. ${ }^{19}$ So, PMB shows relatively better outcomes in bacteremia caused by serious MDR 
gram-negative bacteria such as, CR- $K p$ and our current study also found a comprehensive better result in middle aged patients with PMB with least number of secondary infection in comparison to CLN.

The pathogenic characteristics and spreading tendency of bacteria in the bloodstream is not always same, and dissemination of the virulent pathogen to different tissues are very common. ${ }^{8}$ A research on animal models showed that bloodstream is a major route of spreading infectious pathogens to remote tissues from the primary site of infection and development of bacteremia consequently, increases the chances of rapid dissemination of that virulent pathogen to surrounding tissue..$^{20,21}$ So, appropriate antibiotic is required in the beginning of the bacteremia treatment to minimize the rate of further secondary infections and our study demonstrated the relatively less quantity of secondary infection in PMB group (4 cases; lung) than the CLN group (15 cases; lung and wound swab).

Mortality rate in CR- $\mathrm{Kp}$-associated bacteremia is high in hospital settings, globally. A study showed that $71.90 \%$ crude mortality rate is due to CR-Kpassociated bacteremia. ${ }^{23}$ A $47 \%$ 14-day mortality was estimated with CR-Kp-associated BSI in a New York based outbreak report and rate of rehospitalization was so high. ${ }^{24}$ Similarly, in CR-Kp bacteremia, our study found a higher 30 -day mortality rate (17.37\%) in CLN group in comparison to the PMB group. This report significantly indicates the treatmentsuperiority of polymyxin B in CR- $K p$ bacteremia, whereas multiple studies observed similar type of superiority of colistin in CR-Kp-associated urinary tract infections. ${ }^{14,15}$

The major limitation of this study is that it did not observe the long term (>1 month) outcomes of PMB and CLN therapies in combination with other possible antibiotics in CR- $K p$-associated bacteremia with other co-morbidities. Further mega studies are required to evaluate the relatively better beneficial role of PMB and CLN against CR-Kp-associated different type of infections.

\section{CONCLUSION}

CR-Kp-associated bacteremia is a life threatening infection with few potential antibiotictreatment options including polymyxins. So site of infection oriented superiority of antibiotic therapies concerning the relative therapeutic outcomes and least mortality rate is ideal to consider during this emergence of superbugs. In this study, polymyxin B showed better therapeutic outcomes than colistin in the CR- $K p$-associated bacteremia and may be considered as the first-line treatment option.

\section{Conflicts of interest}

No conflicts of interest.

\section{ACKNOWLEDGEMENT}

All authors of this study are very grateful to all ICU doctors and authority of Square hospital because of their permission for this study and all kind of cooperation as well as time to time supports during the study.

\section{REFERENCES}

1. Storm, D.R., Rosenthal, K.S. and Swanson, P.E. 1977. Polymyxin and related peptide antibiotics. Annu. Rev. Biochem. 46, 723-163.

2. Gupta, S., Govil, D., Kakar, P.N., Prakash, O., Arora. D., Das, S., Govil, P. and Malhotra, A. 2009. Colistin and polymyxin B: a re-emergence. Indian J. Crit. Care Med. 13, 49-53.

3. Newton, B.A. 1956. The properties and mode of action of the polymyxins. Bacteriol. Rev. 20, 14-27.

4. Venkatachalam, I., Teo, J., Balm, M.N., Fisher, D.A., Jureen, R. and Lin, R.T. 2012. Klebsiella pneumoniae Carbapenemase-producing Enterobacteria in Hospital, Singapore. Emerg. Infect. Dis. 18, 1381-1383.

5. Picão, R.C., Jones, R.N., Mendes, R.E. and Castanheira, M. 2013. Klebsiella pneumoniae carbapenemase-producing enterobacteriaceae testing susceptible to cefepime by reference methods. J. Clin. Microbiol. 51, 2388-2390.

6. World Health Organization. Antibiotic resistance. Available at: https://www.who.int/news-room/fact-sheets/detail/antimicrobial-resistance. Accessed November 30, 2018. 
7. Centers for Disease Control and Prevention, US Department of Health and Human Services. 2013. Antibiotic resistance threats in the United States, 2013. Available at: https://www.cdc.gov/drugresistance/pdf/ar-threats-2013508.pdf. Accessed December 10, 2018.

8. Christaki, E. and Giamarellos-Bourboulis, E.J. 2014. The complex pathogenesis of bacteremia: from antimicrobial clearance mechanisms to the genetic background of the host. Virulence 5, 57-65.

9. Neuner, E.A., Yeh, J.Y., Hall, G.S., Sekeres, J., Endimiani, A., Bonomo, R.A., Shrestha, N.K., Fraser, T.G. and van, Duin. D. 2011. Treatment and outcomes in carbapenemresistant Klebsiella pneumoniae bloodstream infections. Diagn. Microbiol. Infect. Dis. 69, 357-362.

10. Hirsch, E.B. and Tam, V.H. 2010. Detection and treatment options for Klebsiella pneumoniae carbapenemases (KPCs): an emerging cause of multidrug-resistant infection. $J$. Antimicrob. Chemother. 65, 1119-1125.

11. Gales, A.C., Jones, R.N. and Sader, H.S. 2011. Contemporary activity of colistin and polymyxin B against a worldwide collection of Gram-negative pathogens: results from the SENTRY Antimicrobial Surveillance Program (2006-09). J. Antimicrob. Chemother. 66, 2070-2074.

12. Kumar, A. and Singh, N.P. 2015. Antimicrobial dosing in critically ill patients with sepsis-induced acute kidney injury. Indian J. Crit. Care Med. 19, 99-108.

13. Poirel, L., Jayol, A. and Nordmann, P. 2017. Polymyxins: antibacterial activity, susceptibility testing, and resistance mechanisms encoded by plasmids or chromosomes. Clin. Microbiol. Rev. 30, 557-596.

14. Garonzik, S.M., Li, J., Thamlikitkul, V., Paterson, D.L., Shoham, S., Jacob, J., Silveira, F.P., Forrest, A. and Nation, R.L. 2011. Population pharmacokinetics of colistin methanesulfonate and formed colistin in critically ill patients from a multicenter study provide dosing suggestions for various categories of patients. Antimicrob. Agents Chemother. 55, 3284-3294.

15. Plachouras, D., Karvanen, M., Friberg, L.E., Papadomichelakis, E., Antoniadou, A., Tsangaris, I., Karaiskos, I., Poulakou, G., Kontopidou, F., Armaganidis, A., Cars, O. and Giamarellou, H. 2009. Population pharmacokinetic analysis of colistin methanesul-phonate and colistin after intravenous administration in critically ill patients with infections caused by Gram-negative bacteria. Antimicrob. Agents Chemother. 53, 3430-3436.

16. Sandri, A.M., Landersdorfer, C.B., Jacob, J., Boniatti, M.M., Dalarosa, M.G., Falci, D.R., Behle, T.F., Bordinhão, R.C., Wang, J., Forrest, A., Nation, R.L., Li, J. and Zavascki, A.P. 2013. Population pharmacokinetics of polymyxin B in critically ill patients: Implica-tions for selection of dosage regimens. Clin. Infect. Dis. 57, 524-531.
17. Sandri, A.M., Landersdorfer, C.B., Jacob, J., Boniatti, M.M., Dalarosa, M.G., Falci, D.R., Behle, T.F., Saitovitch, D., Wang, J., Forrest, A., Nation, R.L., Zavascki, A.P. and Li, J. 2013. Pharmacokinetics of polymyxin B in patients on continuous venovenous haemodialysis. J. Antimicrob. Chemother. 68, 674-677.

18. Ngamprasertchai, T., Boonyasiri, A., Charoenpong, L., Nimitvilai, S., Lorchirachoonkul, N., Wattanamongkonsi,1 L. and Thamlikitkul, V. 2018. Effectiveness and safety of polymyxin B for the treatment of infections caused by extensively drug-resistant Gram-negative bacteria in Thailand. Infect. Drug. Resist. 1, 1219-1224.

19. Karakkattu, J., Mohan, A., James, E. and Kumar, A. 2017. Effectiveness and safety of colistin in multi drug resistant urinary tract infections. J. App. Pharm. Sci. 7, 148-152.

20. Krasnodembskaya, A., Samarani, G., Song, Y., Zhuo, H., Su, X., Lee, J.W., Gupta, N., Petrini, M. and Matthay, M.A. 2012. Human mesenchymal stem cells reduce mortality and bacteremia in gram-negative sepsis in mice in part by enhancing the phagocytic activity of blood monocytes. Am. J. Physiol. Lung Cell. Mol. Physiol. 302, 1003-1013.

21. Giamarellos-Bourboulis, E.J., Adamis, T., Laoutaris, G., Sabracos, L., Koussoulas, V., Mouktaroudi, M., Perrea, D., Karayannacos, P.E. and Giamarellou, H. 2004. Immunomodulatory clarithromycin treatment of experimental sepsis and acute pyelonephritis caused by multidrug-resistant Pseudomonas aeruginosa. Antimicrob. Agents Chemother. 48, 93-99.

22. European Centre for Disease Prevention and Control (ECDC). SURVEILLANCE REPORT, Antimicrobial resistance surveillance in Europe 2013. Annual Report of the European Antimicrobial Resistance Surveillance Network (EARS-Net). Available at: http://ecdc.europa.eu/sites/portal/files/media/en/publications/ Publications/antimicrobial-resistance-surveillance-europe2013.pdf. Accessed December 10, 2018.

23. Borer, A., Saidel-Odes, L., Riesenberg, K., Eskira, S., Peled, N., Nativ, R., Schlaeffer, F. and Sherf, M. 2009. Attributable mortality rate for carbapenem-resistant Klebsiella pneumoniae bacteremia. Infect. Control Hosp. Epidemiol. 30, 972-976.

24. Bratu, S., Landman, D., Haag, R., Recco, R., Eramo, A., Alam, M. and Quale, J. 2005. Rapid spread of carbapenemresistant Klebsiella pneumonia in New York City: a new threat to our antibiotic armamentarium. Arch. Intern. Med. 165, 1430-1435. 